Felipe Ignacio Peña Galaz

Universidad de Santiago de Chile

felipe.pena@usach.cl

\title{
Publicidad Social e Inteligencia territorial en el contexto de la propagación de Didymosphenia geminata en las zonas de Villarrica-Pucón y Panguipulli
}

\author{
Social advertising and territorial inteligence in the context of \\ Didymosphenia geminata propagation in Villarrica-Pucón and \\ Pangupulli zone
}

\begin{abstract}
Resumen
El Didymosphenia Geminata, desde su aparición en Chile en el año 2010, específicamente en la zona de Futaleufú, es monitoreado por SUBPESCA a través de su Fondo de Investigación Pesquero y Acuicultura (Fipa), de este modo capacita y difunde sobre los efectos de la propagación de la microalga Didymo, la que está presente en Chile entre las regiones del Biobío y Magallanes. La plaga ha sido foco de vigilancia, detección y control, junto a campañas de comunicación y publicidad social por parte de SERNAPESCA y organismos privados de consultoría.

El Didymo es una alga que habita en aguas frías continentales, prospera en una amplia gama de condiciones hidráulicas (Kilroy et al. 2005). La literatura científica la describe como una microalga de gran propagación y al hombre como principal vector de transmisión. Localidades de Villarrica, Pucón y Panguipulli, son zonas declaradas con la plaga Didymo en sus ríos. En este escenario, se elaboró un estudio etnográfico a través de la observación en las localidades mencionadas.
\end{abstract}

Palabras Clave: Didymosphenia geminata, Ríos chilenos nordpatagonicos, Inteligencia territorial, Publicidad social.

\begin{abstract}
Since the appearance of Didymosphera Geminata in Chile in 2010, specifically in the Futaleufú zone, is monitored by SUBPESCA, through its Fishing and Aquaculture Investigation Fund (FIPA), thus training and disseminating the effects of Didymo microalgae spread, which is present in the Biobio and Magallanes regions. It has been a focus of surveillance, detection and control, together with communication and social advertising campaigns by SERNAPESCA and private consulting organizations. The Didymo is an algae that lives in cold continental waters, thrives in a wide range of hydraulic conditions (Kilroy et al., 2005). The scientific literature describes it as a microalgae of great propagation and man as the main transmission vector. Localities of Villarrica, Pucón and Panguipulli, are areas
\end{abstract}


declared with the plague Didymo in their rivers. In this scenario, an ethnographic study was prepared through observation in the mentioned locations.

Keywords: Didymosphenia geminata, Chilean rivers Norpatagonicos, Territorial intelligence, Social advertising.

\section{Introducción}

El estudio elaborado en la IX región de la Araucanía, en las localidades de Villarrica y Pucón, y en la XIV región de Los Ríos en la localidad de Panguipulli, tiene como propósito indagar el impacto de las campañas de comunicación y publicidad social (Orozco, 2010) que se diseñaron para estas zonas por parte del Servicio Nacional de Pesca y Acuicultura (SERNAPESCA), organismo dependiente del Ministerio de Economía, Fomento y Turismo, frente al problema por plaga de Didymosphenia geminata (D. Geminata) (Lyngbye) ${ }^{1}$ M. Schmidt $(1899)^{2}$ en los cuerpos de agua de estas regiones. En este contexto y de acuerdo al informe FIP ${ }^{3}$ 2013-25 (SUBPESCA), la Subsecretaría de Pesca y Acuicultura (SUBPESCA) y el Servicio Nacional de Pesca y Acuicultura (SERNAPESCA) han invertido esfuerzos y recursos en la realización de proyectos para la prospección y monitoreo de la plaga, generando campañas de difusión a nivel nacional, manuales de bioseguridad (Díaz et al., 2012) e instalando puntos de desinfección en distintos lugares y pasos fronterizos del sur del país. La aplicación de estas medidas, ha tenido como resultados en las distintas campañas de prospección, vigilancia y monitoreo detectar nuevos focos de plaga en la zona centro-sur y austral del país. Esta investigación busca además responder, si las comunidades y los distintos actores locales están informados o si estos se han involucrado frente al riesgo de esta plaga en el territorio.

\section{La plaga y sus consecuencias}

En síntesis, para entender qué es la microalga Didymo y como se convierte en plaga, Jaramillo (2015) la describe como una microalga de agua dulce. Oliva et al. (2014), indica que

\footnotetext{
${ }^{1}$ Según el estado del arte, esta microalga es descrita inicialmente como Echinella geminata por Lyngbye en 1819. Aproximadamente cinco años después Agardh C. la nombró como Gomphonema geminatum.

${ }^{2}$ En el año 1899 M. Schmidt la clasifica dentro de la familia de las Gomphonemataceae, dándole así su actual nombre (Whitton 2009).

${ }^{3}$ Fondo de Investigación Pesquera y Acuicultura. Ministerio de Economía, Fomento y Turismo (2014).
} 


\section{Felipe Ignacio Peña Galaz}

esta microalga se convierte en plaga, cuando se desprende de su hábitat para adherirse al fitoplancton ${ }^{4}$ que está en las columnas de agua. De esta forma la microalga Didymosphenia geminata prolifera formando cojines mucosos en las riberas de los ríos, contaminado el hábitat normal del caudal, es aquí cuando escoge un lugar que reúne las condiciones fisico-químicas para contaminar y expandirse, en esta etapa se le conoce en la literatura como Didymo o moco de roca.

El informe FIP 2013-25, señala que Didymosphenia geminata prefiere ambientes fríos con pocos nutrientes (oligotróficos), velocidad de flujo moderada, caudales no muy altos, estabilidad de sustrato, alcanza un óptimo crecimiento sobre rocas y bolones en aguas no muy profundas con transparencia, que permita una alta luminosidad (Kilroy, 2004; Kilroy et al., 2005; Larned et al., 2006; Kilroy et al., 2007; Lagerstedt, 2007, SUBPESCA/Amakaik-EcoHyd, 2012). De este modo, D. geminata coloniza más rápidamente sobre rocas ásperas que rocas lisas (Bergey et al. 2009), existen evidencias que ha sido encontrada en cuerpos de agua someras y también profundas. Esta alga encontrada en aguas de los ríos del sur de Chile (Rivera et al. 2013) ha sido considerada como una plaga en fuentes de agua dulce por el Estado de Chile quién genera su plan de manejo ${ }^{5}$ (Reid et al. 2012).

Es así como la contaminación de un río por Didymo provoca consecuencias negativas para las especies acuáticas, la pesca recreativa y el turismo local. A nivel internacional, es justo mencionar a Nueva Zelanda, país con la invasión más comparable al caso chileno debido a sus características ambientales, llegando como especie introducida que ha causado millonarios daños en bioseguridad y turismo ${ }^{6}$. Por lo tanto en Chile, los organismos trabajan para evitar su dispersión a través de acciones medidas por la evidencia científica y la experiencia internacional, indicando al principal vector de dispersión y propagación al hombre (SERNAPESCA, 2012). Los individuos producto de las distintas actividades que realizan en los cuerpos de agua continentales diseminan esta plaga a través del transporte de equipos de pesca recreativa, kayak, botes de

\footnotetext{
${ }^{4}$ El fitoplancton son los seres vivos de origen vegetal que viven flotando en la columna de agua, son organismos capaces de realizar la fotosíntesis. Recuperado de: https://cienciaybiologia.com/fitoplancton/

${ }^{5}$ Según Res. Ex. 1866 (SUBPESCA, 2015).

${ }^{6}$ El Mostrador, Agenda País 2030, 2017.
} 


\section{Felipe Ignacio Peña Galaz}

rafting, equipos de trekking, entre otros artículos, los cuales tuvieron contacto con ríos o lagos con plaga Didymo.

Es escasa la información acerca de la plaga Didymo en Chile, por el momento la microalga no tiene efectos nocivos o produce muertes en humanos, por lo cual el impacto mediático no ha sido de gran envergadura en el curso de este problema aun cuando se ha informado a los medios. Por el momento, no existen centros de investigación que aborden el problema en su realidad cultural y local.

El efecto económico de la plaga Didymo está lejos de ser calculado en Chile, ya que las medidas de control existentes también podrían tener efectos en la industria del turismo y la acuicultura, donde la inversión pública en este contexto es menor y escasa.

\section{Propagación por Didymosphenia geminata en Chile}

Desde la década de los noventa, el territorio Chileno tuvo un relevante e importante incremento de turistas, gracias al impulso de los recursos hídricos que se puede explotar bajo el sistema mercantilista que Chile posee (Herrera, 2012). Constanza Herrera señala:

"La importancia radica principalmente en el sector económico que se ve potenciado por las actividades extractivas y los atractivos turísticos que cada año estimulan a más extranjeros a visitar el país por su extraordinaria belleza y abundante biodiversidad acuática". (2012:1)

La IX Región y las zonas sur-austral de Chile, se vuelven un importante atractivo para practicar la pesca recreativa y deportiva, la cual se vuelve un atractivo turístico muy importante gracias a lo relevante de las cuencas y la diversidad de poblaciones acuáticas que en ellas habitan. Estas prácticas que dejan importantes ingresos para las regiones y el país, a la vez implican un deterioro importante del medio ambiente. La controversia que nace aquí es producto de esta explotación.

Las zonas de la IX región de la Araucanía y la región XIV de Los Ríos, poseen una diversidad considerable para la explotación de sus recursos naturales y prácticas de riesgo para el 
ecosistema. El impacto económico de esta plaga aún es desconocido, pero se puede estimar por ejemplo gracias a un estudio de la Universidad Arturo Prat del año 2007, donde sólo la práctica de pesca recreativa representa un ingreso promedio de US\$34 millones anuales. Ya para el 2002, el turismo en Chile representaba el quinto lugar de aporte al PIB nacional, por arriba sobre la exportación de Salmón, donde el turismo dejaba cifras de unos US\$827,9 millones, sólo por debajo de los productos de exportación forestal (INE, 2002). No obstante, según la Balanza de Pagos del Banco Central de Chile durante el 2013, arrojaba cifras para la exportación del Salmón por US\$2.771,8 en relación al Turismo Nacional de US\$2.581,1 (SERNATUR, 2014-2018).

Para el 2016, los Parques Nacional de la zona de Villarrica y Pucón reciben unos 70.832 visitantes, siendo la región de la Araucanía, la sexta en el ranking más visitada del país (SERNATUR, 2016). La erradicación de Didymo no se ha podido demostrar en Chile y ningún país. Se desconocen las secuelas a corto y largo plazo (Herrera, 2012). Cuando hay presencia, el biosistema aumenta y se reduce la disponibilidad de oxígeno en los cuerpos de agua, modificando la concentración de nutrientes que reducirían fuertemente, la biodiversidad y la población de especies, ya que se está alterando el hábitat y ecosistema existente, perjudicando los ingresos para estas regiones.

La ciudad de Pucón es un lugar de alta demanda turística y de un importante circuito deportivo como es el caso del "Ironman de Pucón”, uno de los más importante eventos deportivos en Chile. Esta actividad puede dejar cifras cercanas a los US\$ 5 millones en ganancias en relación al total de la actividad turística nacional, aportando de manera significativa a la generación de empleo en la zona y al PIB nacional. Este tipo de actividades pudiera verse fuertemente afectado en el desarrollo futuro, con un gran impacto ambiental si no se tiene por objeto el desarrollo sostenible y por sujeto a la comunidad territorial la cual debe involucrarse en la prospección del entorno. Es así como el Estado ha financiado para investigación, desarrollo y difusión, ya que si esto no es abordado de forma estratégica a la ciudadanía, quién a la vez visita estas macrozonas en gran cantidad y de forma esporádica, tendremos científicos, que según Hannigan (2006) no solo hacen afirmaciones de conocimiento, sino que también construyen rutinariamente "ignorancia".

Hannigan, citando a Aronson (1984) señala la existencia de tres tipos de afirmaciones interpretativas que hacen los científicos: problema técnico, cultural y social. Según Broitman y 
Kreimer (2017) hace décadas que los estudios sociales de la ciencia se han interesado en las controversias socio-científicas. Producto de la explotación de recursos, donde no se han tomado las medidas y resguardos correspondientes, nace la presencia de agentes introducidos, como es el caso de Didymo, una especie altamente invasora de difícil erradicación, por no decir imposible (Herrera, 2012).

El Didymo es una diatomea bentónica o alga que prospera en una amplia gama de condiciones hidráulicas (Kilroy et al. 2005), se sabe que puede prosperar en una amplia variedad de condiciones fisicoquímicas en ríos y lagos, y que puede ser trasportada accidentalmente por el hombre a través de indumentaria y equipos, lo que la transforma en una seria amenaza para los ecosistemas acuáticos alrededor del mundo. Los ríos en el hemisferio sur están especialmente en riesgo de nuevas introducciones y dispersiones dentro de las áreas ya afectadas, en particular distintas cuencas de Australia, Argentina, Perú y Chile.

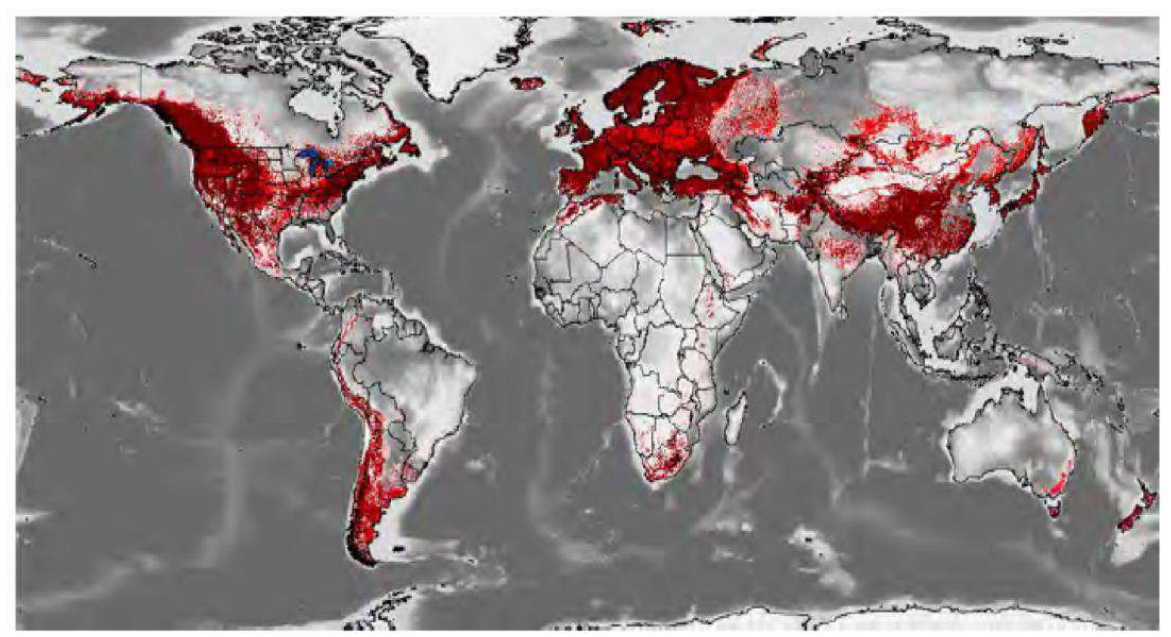

Figura 1: Didymosphenia geminata (Lyngbye) Mart.Schmidt. Hábitat potencial.

Fuente: Increase in nuisance blooms and geographic expansion of the freshwater diatom Didymosphenia geminate (2007).

Desde su aparición en Chile, el Estado ha invertido más de $\$ 500.000 .000{ }^{8}$ en investigación, material de difusión y contención de esta plaga. De este modo, la Subsecretaría de Pesca y Acuicultura (SUBPESCA) a través de SERNAPESCA, en conjunto a consultorías

\footnotetext{
${ }^{7}$ Recuperado de: $\mathrm{http} / / /$ sernapesca/menu_que_es_didymo/problema.php

${ }^{8}$ Recuperado de: http://www.conicyt.cl/regional/2014/07/17/centro-regional-cequa-se-adjudica-importantefinanciamiento-para-combatir-propagacion-del-didymo/
} 
ambientales como Fundación Cequa, Amakaik, Ecohyd, IFOP, POCH ambiental, entre otras, han trabajado bajo asignaciones y presupuestos concursables del Estado para la prospección de esta microalga y sus efectos en el territorio.

Se especula que la constante presencia de pescadores deportivos extranjeros pudo haber constribuido a la aparición de D. Geminata. Estos turistas no son una cifra considerable, pero en periodos de pesca contribuyen paulatinamente a contaminar, sobre todo, porque la misma riqueza de la fauna marina y el ecosistema que rodea los ríos del sur de Chile (Herrera, 2012), está en pleno desarrollo.

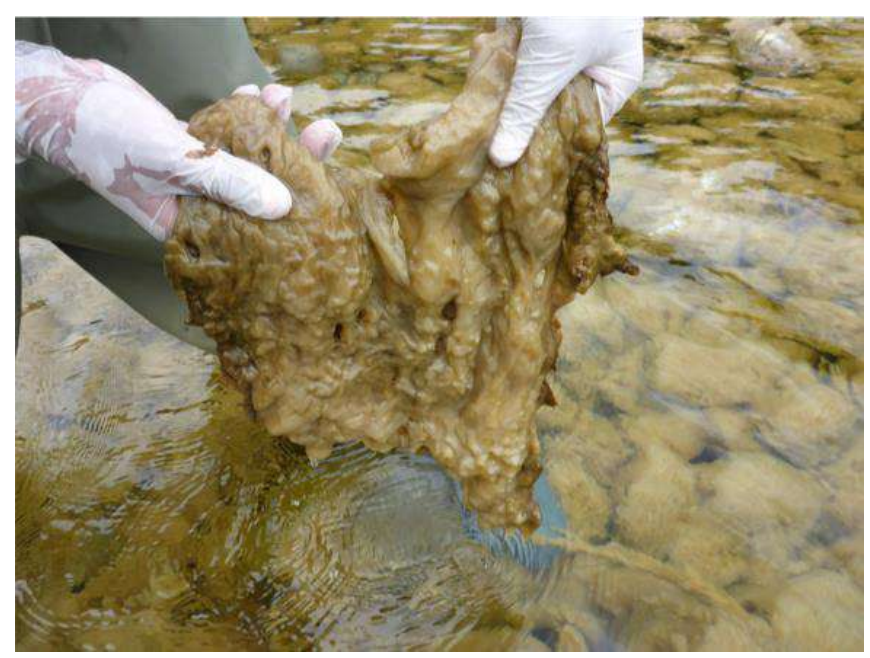

Figura 2: Moco de roca o D. geminata.

Fuente: Salvo, J., Oyanedel, A. IFOP-SUBPESCA (2017).

Frente a este problema de plaga, SERNAPESCA ha ocupado millonarios recursos y ha difundido material de los resultados de investigaciones a organismos especializados en la materia, también a colegios rurales, ONG's, municipalidades, centros comunitarios, donde se han elaborado cursos, talleres y seminarios para la capacitación y divulgación en cuanto refiere a Didymo, la limpieza y contención de este.

El D. Geminata, presente en Chile entre las regiones del Biobío y Magallanes, ha sido foco de vigilancia, detección y control desde su aparición en la zona de Futaleufú. La Subsecretaría de Pesca y Acuicultura (SUBPESCA), a través de su Fondo de Investigación Pesquero y Acuicultura (Fipa), capacita y difunde a servicios públicos y personas naturales sobre 
la realidad y los efectos de la propagación de Didymo, utilizando las bases del "Manual para el monitoreo de identificación de la microalga bentónica Didymosphenia geminata", (Salmonexpert, 2016).

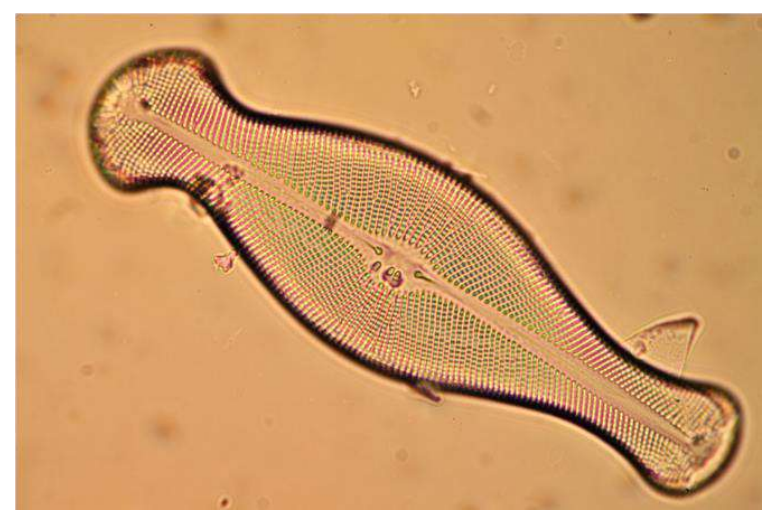

Figura 3: Microscopica Didymosphenia geminata (Lyngbye) Mart. Schmidt.

Fuente: Salvo, J., Oyanedel, A. IFOP-SUBPESCA (2017).

En este escenario, las microalgas encuentran variables físico-químicas que son apropiadas para la dispersión. Los investigadores trabajan en el desarrollo de métodos y/o protocolos que permitan minimizar, evaluar y mitigar su propagación, a pesar de estos estudios modernos de recolección de muestras para identificación de especies mediante rasgos morfológicos (Rivera et al. 2013), y la caracterización física, química y biológica de las cuencas hidrográficas impactadas (Díaz et al. 2012) y la transferencia de metodologías de prevención de la invasión (uso de detergentes) que han sido diseñadas en Nueva Zelandia (Jaramillo, 2015), las personas en general desconocen la existencia y el impacto de esta microalga.

\section{Acerca de las campañas de comunicación social por Didymosphenia geminata en Chile}

Para evidenciar cuáles han sido las medidas para contener el Didymo por parte del Estado en las zonas de Villarrica, Pucón y Panguipulli, se determinó visitar estas macrozonas y mediante la observación y el registro fotográfico se pudo determinar que SERNAPESCA ha trabajado distintas campañas de publicidad social y de ese modo evitar aún más la propagación. Estas 


\section{Felipe Ignacio Peña Galaz}

campañas de comunicación e imagen y difusión han sido elaboradas por el mismo Servicio Nacional de Pesca y Acuicultura (SERNAPESCA).

El organismo SERNAPESCA a través de su sitio web institucional, se define como el "Servicio Nacional de Pesca y Acuicultura", entidad dependiente del Ministerio de Economía, Fomento y Turismo, cuya misión es contribuir a la sustentabilidad del sector y a la protección de los recursos hidrobiológicos y su medio ambiente, a través de una fiscalización integral y gestión sanitaria que influye en el comportamiento sectorial promoviendo el cumplimiento de las normas. Por la dinámica propia del sector, requiere de la coordinación y trabajos conjuntos con organizaciones públicas y privadas, tanto nacionales como internacionales y diversos centros de estudios.

Según datos extraídos a personal de fiscalización de SERNAPESCA, con sede en Temuco, donde además facilitaron archivos digitales de piezas gráficas de sus distintas campañas, expresaron que el equipo de comunicaciones del organismo ha tomado siempre la responsabilidad de diseñar las distintas estrategias comunicacionales, de ese modo para el año 2011, elaboraron la primera campaña comunicacional bajo el slogan “ALTO AL DIDYMO”, en lenguaje Español e Inglés; se diseñaron piezas gráficas con tipografías en mayúsculas. En términos semiológicos, adquieren los soportes gráficos, un mensaje claro, directo y fácil de comprender, siendo el concepto central el texto "ALTO AL DIDYMO", como lo muestra la figura 4, acompañado de la misma forma tipográfica "REMUEVA, LAVE y SEQUE", junto a fondos de color primarios rojo y azul. Además, se utilizaron fotografías alusivas a ríos del sur de Chile e imágenes de moco de roca, lo cual representó la forma física de esta plaga. Esta campaña a través de estas piezas gráficas si bien funciona con claridad, el desconocimiento aún está presente en los individuos y las comunidades locales.

Es así, como SERNAPESCA y el Instituto Santo Tomás Sede Temuco, trabajaron durante los años 2014 y 2015 junto a estudiantes de la Escuela de Diseño, en el desarrollo de una nueva campaña comunicacional para el territorio, cuyo concepto central fue "EN ESTE ECOSISTEMA, EL DIDYMO NO ENCAJA”, slogan que fue impulsado por SERNAPESCA, campaña aplicada en distintas piezas gráficas en torno a la plaga. Ya que los alumnos de la Escuela de Diseño que participaron de esta convocatoria manejaban poca información al respecto, los alumnos expresaron a medios de comunicación locales "Francamente, conocíamos poco y nada al 
respecto", por lo cual, se realizó un proceso creativo bajo la supervisión por parte de encargados de la campaña del organismo SERNAPESCA, diseñadores externos contratados para la ocasión y distintos actores del Instituto.

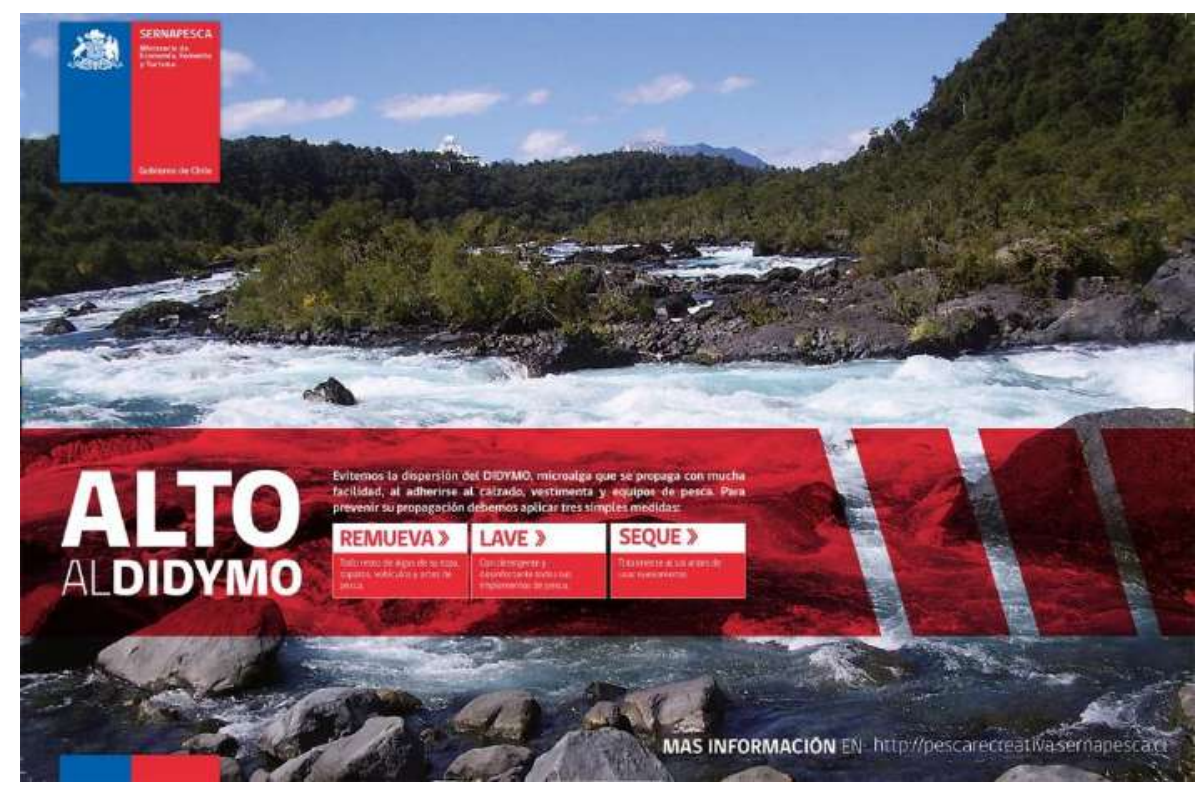

Figura 4: Lienzo campaña difusión 2011.

Fuente: Pieza gráfica, gentileza SERNAPESCA, Sede Temuco.

Durante estos años de publicidad social en el contexto de plaga, la forma de representar el Didymo en las distintas campañas de difusión que ha elaborado SERNAPESCA (como mandante) junto a los distintos actores que han apoyado y aportado en esta labor, ha sido a través de piezas gráficas con abundancia ilustrativa, junto a personajes e íconos lúdicos orientadas a la familia. Joan Costa, describe que la abundante información expuesta en las piezas gráficas que apoyan la publicidad social, son un problema de sobrecarga de información visual, el cual denomina como "lastre" donde él señala:

"Las ilustraciones que darían origen a su desarrollo como marcas fueron perdiendo así los detalles, los elementos accesorios, los rasgos secundarios. El exceso de realismo y de detalles superfluos, la superposición de elementos, referencias y argumentos en estos antiguos mensajes, fueron considerándose como "lastres" que irían soltándose progresivamente como 
una sobrecarga, en busca de una mayor funcionalidad una expresividad más directa" (Costa, 2004: 86).

Las palabras REMUEVA, LAVE y SEQUE ${ }^{9}$, son los términos principales de las normativas y resoluciones establecidas para la contención de Didymo por parte del Estado de Chile gracias a la experiencia internacional y estudios científicos, términos que han sido utilizados de forma secundarias por SERNAPESCA y los organismos colaborativos en las publicidades sociales, ya que las campañas siempre han utilizado títulos o textos principales como por ejemplo "ALTO AL DIDYMO", "EN ESTE ECOSISTEMA, EL DIDYMO NO ENCAJA" o "HAZLE CASO A LA NORMA".

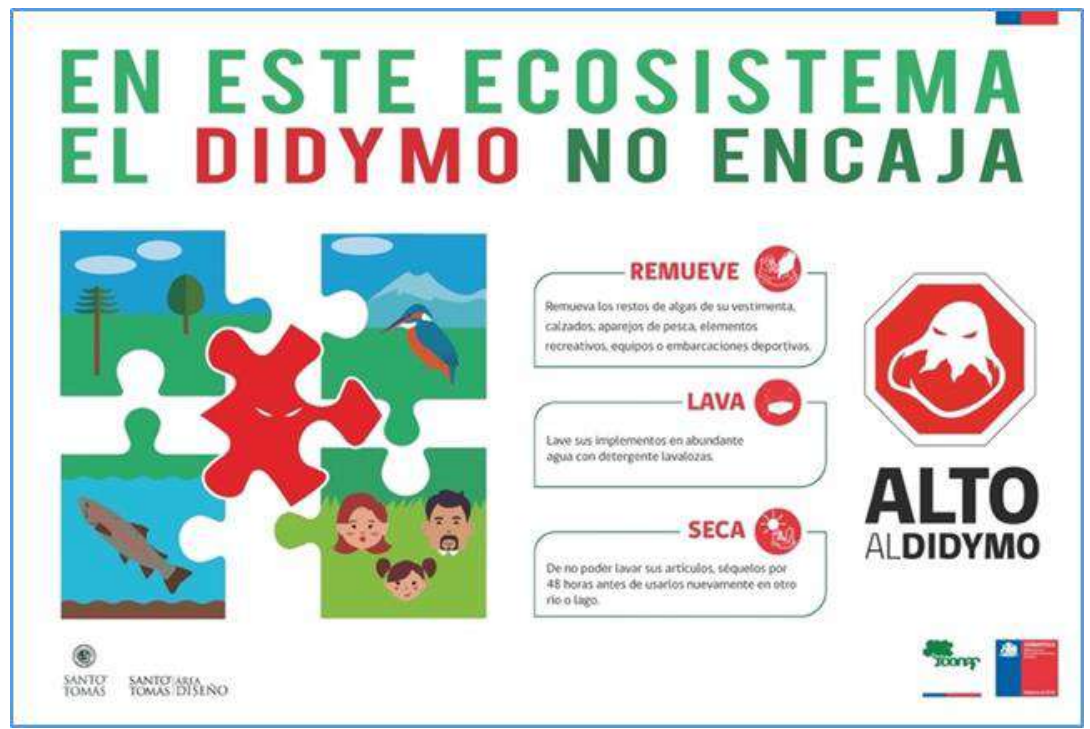

Figura 5: Lienzo campaña difusión 2015-2016.

Fuente: Pieza gráfica, gentileza SERNAPESCA, Sede Temuco.

\footnotetext{
${ }^{9}$ Recuperado de: http://didymo.sernapesca.cl/medidas.php
} 


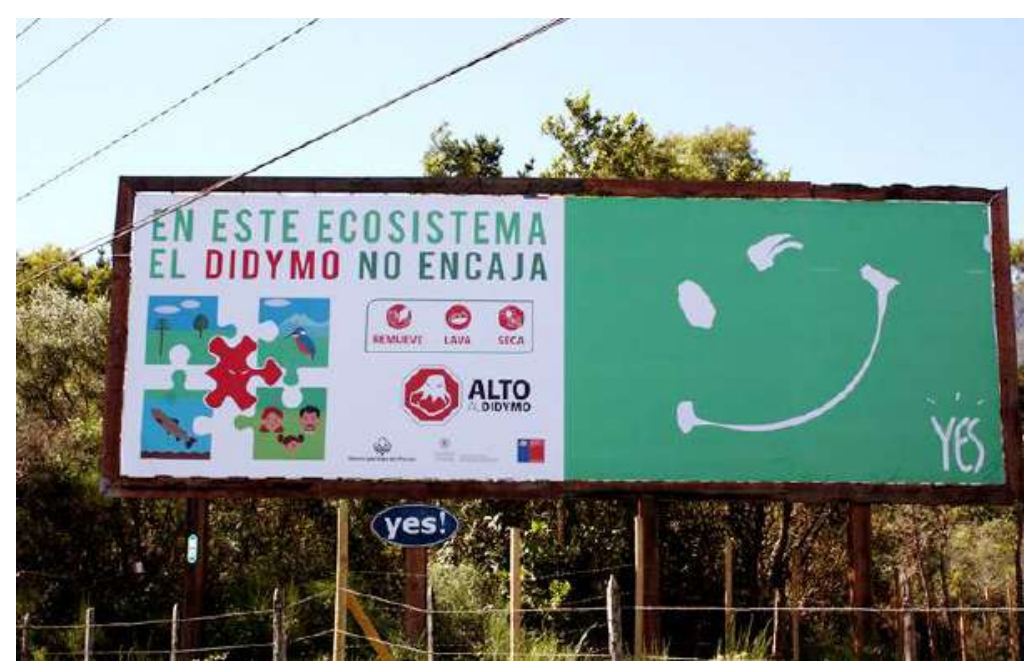

Figura 6: Lienzo campaña difusión 2015-2016, aplicación en vía pública.

Fuente: http://enlinea.santotomas.cl/wp-content/uploads/sites/2/2016/01/campana-didymo.jpg

Durante los períodos 2016 a 2018, el equipo de comunicaciones de SERNAPESCA de Concepción, junto a la Escuela de Diseño del Instituto Santo Tomás Sede Temuco, elaboraron la nueva Campaña de Comunicación y Publicidad Social bajo el concepto "HAZLE CASO A LA NORMA" como muestra la figura 7, la cual mantiene la ilustración como medio de representación gráfica para todas las piezas elaboradas en esta Campaña.

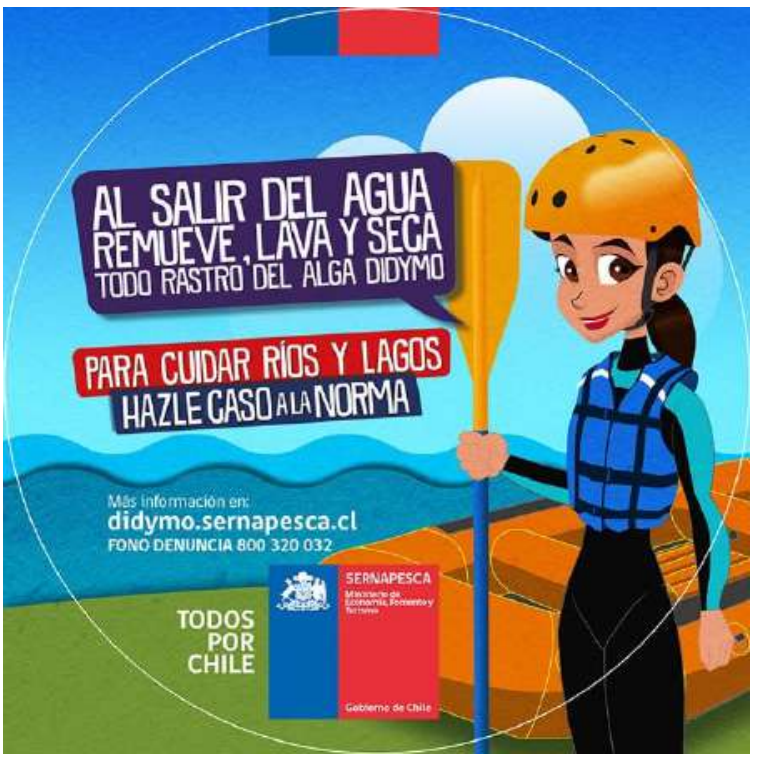

Figura 7: Afiche campaña difusión 2016-2018.

Fuente: Pieza gráfica, gentileza SERNAPESCA, Sede Temuco. 
De este modo la campaña actual (2016-2018) entorno a Didymo "HAZLE CASO A LA NORMA", a simple vista no cumple con el propósito de informar, educar y persuadir a los individuos de las zonas locales y los visitantes esporádicos que hacen uso del río y lagos en el contexto de limpieza, remoción y contención de la plaga, producto del exceso de información, adornos en las piezas gráficas y una orientación del slogan de difícil comprensión.

Si hablamos del diseño de las piezas gráficas, ciertamente el embellecimiento que está fuertemente asociado a este término, es sólo una parte, el diseño es infinitamente más que eso, el diseño no es sólo un adorno. Para apoyar esta descripción, Wong ejemplifica:

"La silla bien diseñada no sólo posee una apariencia exterior agradable, sino que se mantiene firme sobre el piso y da un confort adecuado a quien se sienta en ella. Además debe ser segura y bastante duradera, puede ser producida a un coste comparativamente económico, puede ser embalada y despachada en forma adecuada y, desde luego, debe cumplir una función específica, sea para trabajar, para descansar, para comer o para otras actividades humanas" (Wong 2002: 41)

Para la publicidad social, cualquier interpretación debe ser tan funcional como la silla expuesta por Wong (2002), este ejemplo pone especial cuidado en que, lo que comunique toda campaña de tipo social sea tan claro y transparente que no deje espacio para las malas interpretaciones, que es lo que ocurre comúnmente. Los mensajes con contenidos ambiguos o "muy elevados" "sobrecargados", requieren de una capacidad de comprensión y de análisis que, en ocasiones, no están al alcance de las personas a las que se dirigen (Orozco ,2010).

Según lo evidenciado en el trabajo de campo, la administración de las publicidades acerca de la campaña social "HAZLE CASO A LA NORMA" y los equipos de limpieza que la componen, han sido aplicados en distintos lugares territoriales sin tener una concepción clara a nivel estratégico por parte de SERNAPESCA, como por ejemplo, aplicación de estudios etnográficos en las comunidades afectadas por la plaga, por lo cual, carecen de mantención de elementos físicos por parte del organismo o trabajos de publicidad social bajo un concepto metodológico arraigado a las mismas comunidades, es probable una insuficiente inyección de 
recursos para el mismo propósito, por lo mismo, carecen de una aplicación en soportes adecuados para el fácil acceso de los actores individuos.

El enérgico slogan utilizado por SERNAPESCA "REMUEVA, LAVE y SEQUE", extraído de la experiencia Internacional como "CHECK, CLEAN, DRY" del Biosecurity New Zealand $^{10}$, no debe ser aplicado bajo elementos distractivos en las campañas en Chile, ya sea junto a ilustraciones rodeadas de elementos significantes, personajes y textos que no ayudan en la entrega expedita de información a las personas, olvidando que, el factor humano es el principal vector de propagación de D. geminata. Este slogan, que a su vez funciona como un logotipo, una representación gráfica de una marca que se compone sólo de letras o tipografía, el protagonista principal y no secundario puesto que se percibe y entiende en cuestión de segundos. "REMUEVA, LAVE y SEQUE", aún si carece de un elemento pictórico que las acompañe, es comprendido. Estos textos no son acompañados de un elemento representativo perfectamente iconizado y sintetizado para formar y unificar un logo de gran impacto visual, el cual se pueda convertir en una marca-producto que el individuo capte de un vistazo y que tiene que quedarse fijado en su memoria (Costa, 2004), es así como todos los elementos de sobrecarga que el autor Costa (2004) define como lastre, deben ser eliminados para lograr el mensaje más directo hacia el usuario.

El diseño es un proceso de creación visual con un propósito que viene a apoyar a las campañas de difusión, ya sean comerciales o sociales. A diferencia de la pintura como tal y de la escultura, que son la realización de las visiones personales y los sueños de un artista, el diseño cubre exigencias prácticas. Una unidad de diseño debe ser entonces colocada frente a los ojos del público y transportar de inmediato un mensaje prefijado. Un producto industrial debe cubrir de este mismo modo las necesidades de un consumidor (Wong, 2002).

Un buen diseño para ser aplicado como un elemento de gran relevancia junto a la publicidad social, es la mejor expresión visual de la esencia de "algo", ya sea esto un mensaje o un producto, según Wong:

"se debe buscar la mejor forma posible para que ese "algo" sea conformado, fabricado, distribuido, usado y relacionado con el ambiente asociado. Su

${ }^{10}$ Recuperado de: http://www.mpi.govt.nz/travel-and-recreation/outdoor-activities/check-clean-dry/ 
creación no debe ser sólo estética sino también funcional, mientras refleja o guía el gusto de su época” (Wong 2002: 41)

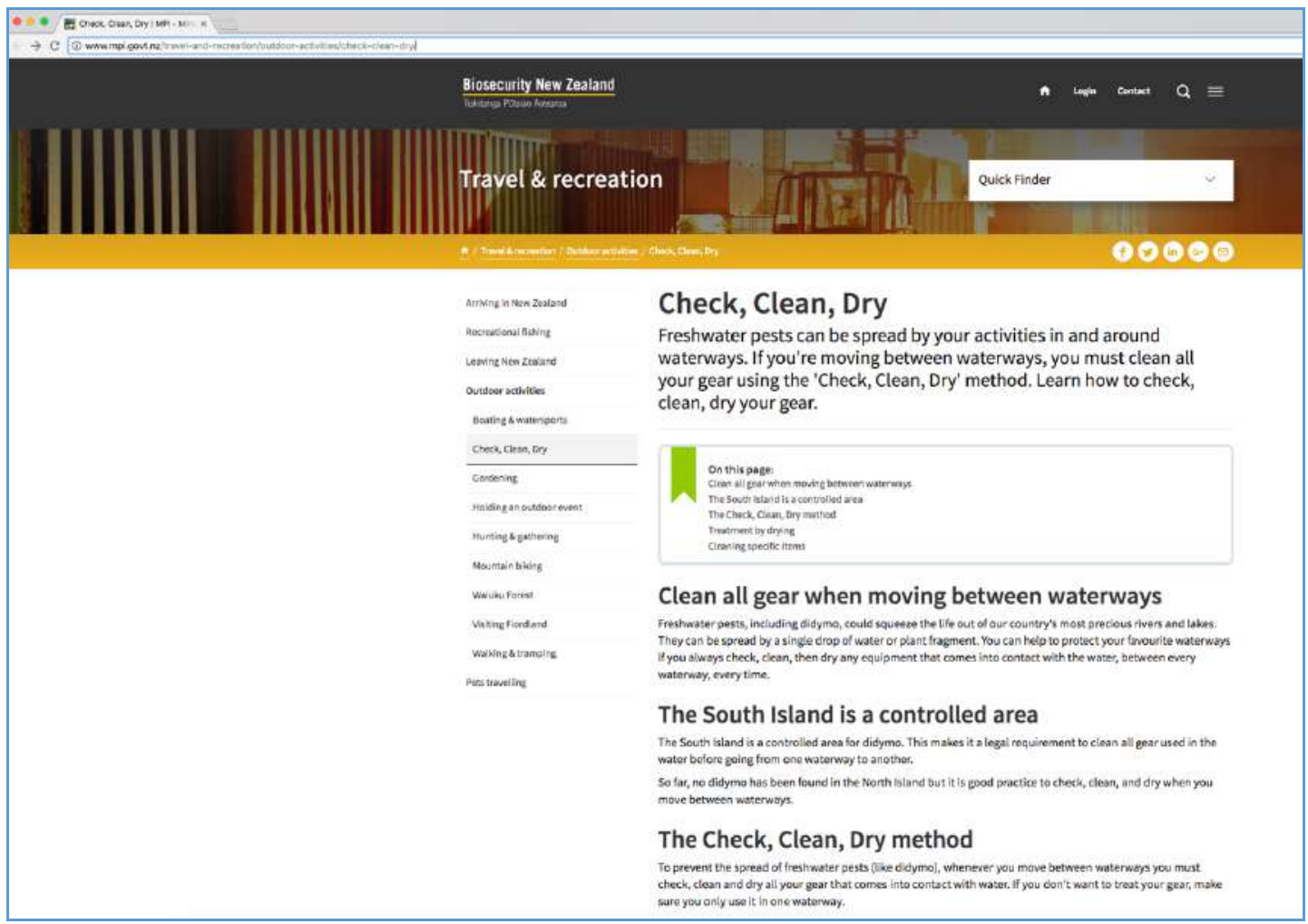

Figura 8: Slogan “Check, Clean, Dry”, Biosecurity New Zealand. Aplicación sitio web official.

Fuente: http://www.mpi.govt.nz/travel-and-recreation/outdoor-activities/check-clean-dry/

El desconocimiento, la poca información y lo confuso que aún es Didymo en la publicidad social en Chile, propicia que los individuos no se involucren en el entorno, en efecto, la microalga gana terreno y sigue expandiéndose por el sur y extremo sur del país, con excelentes condiciones ambientales que aportan aún más en su línea de invasión.

\section{Didymosphenia geminata en Villarrica-Pucón y Panguipulli}

En la Provincia de Cautín, IX Región de La Araucanía, se encuentra la ciudad-comuna de Villarrica, junto a las municipalidades de Gorbea, Cunco, Loncoche, Pucón, Curarrehue y Toltén. La ciudad de Villarrica se emplaza a orillas del lago que lleva el mismo nombre. Por otra parte, el 
territorio de Pucón se localiza en el extremo sureste de la IX Región de la Araucanía. Limita al norte con las comunas de Melipeuco y Cunco, al oeste con Villarrica, al este con Curarrehue y al sur con Panguipulli (XIV Región de Los Ríos), Capital Valdivia.

En la zona del Lago Villarrica, desemboca en la costa de Pucón el río más importante y extenso que alimenta al lago, el río Pucón, con una extensión de $78 \mathrm{~km}$. de longitud. Este río nace en la Laguna Quillelhue, el río inicialmente en el alto río se denomina río Trancura, con afluentes en la zona baja como el río Turbio y el río Liucura que lo intersectan. El río Pucón es un conocido río para la práctica de Ráfting y Kayak por sus importantes rápidos. Además en el río se práctica pesca con mosca, por lo cual frente a estas actividades recreativas, es un importante foco de dispersión y propagación de Didymosphenia geminata. Dada la reciente detección de $D$. geminata en la zona, la subcuenca río Pucón entre río Curileufu y desembocadura lago Villarrica son declaradas áreas de plaga, para el 2012, según la Resolución Exenta No 2634, mediante un informe de la Consultoría Ambiental AMAKAYK y SERNAPESCA región de la Araucanía.

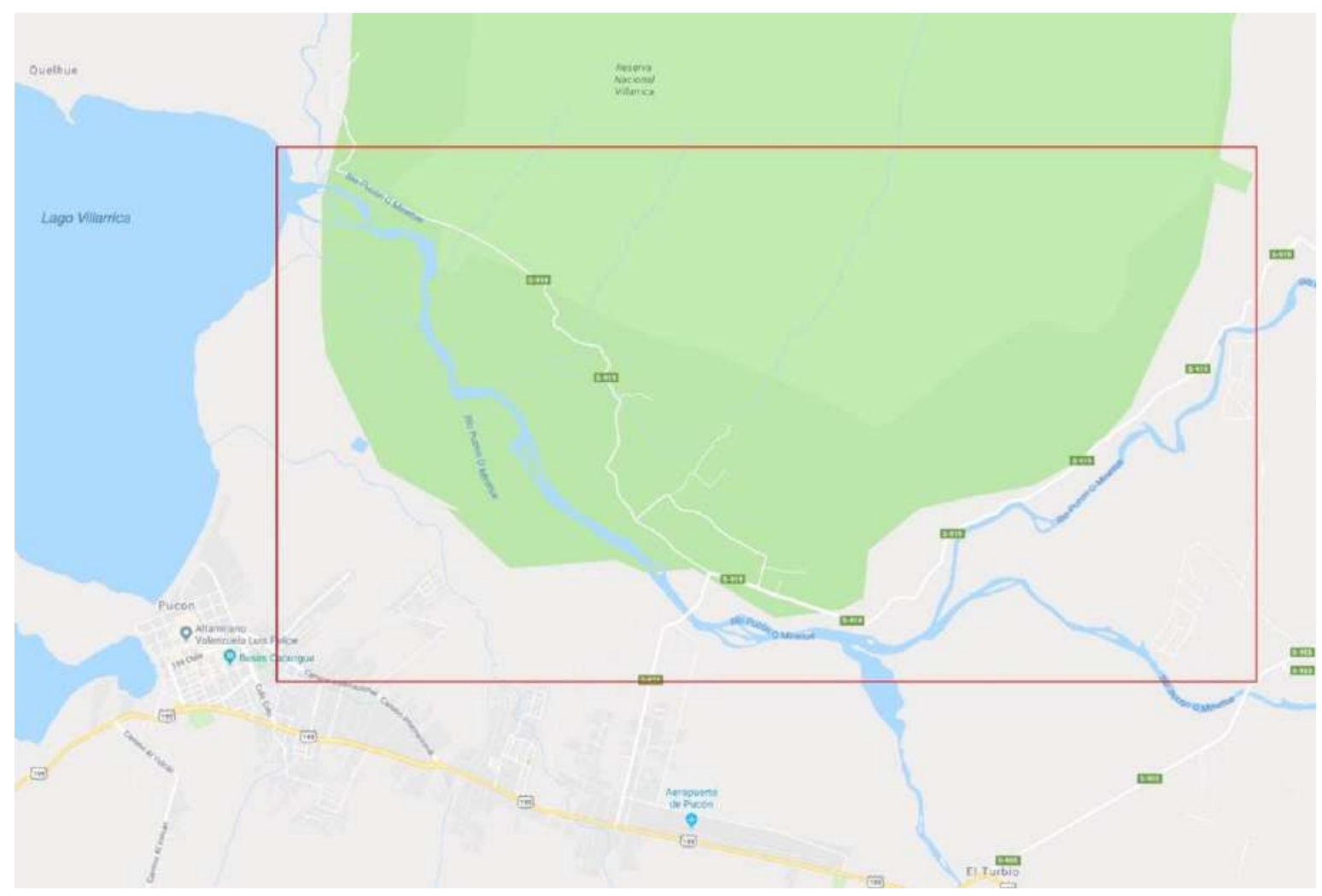

Figura 9: Área geográfica desembocadura (Lago Villarrica) de río Pucón. Demarcación zona de plaga.

Fuente: Google Maps, https://goo.gl/maps/wNuqK5ixCdH2 
La Comuna de Panguipulli, se ubica en la zona de los Siete Lagos, al noreste de la provincia de Valdivia, en la XIV Región de Los Ríos, en este ámbito, esta comuna limita al norte con la comuna de Villarrica, al Oeste con las comunas de Lanco y Máfil, al este y sureste con la república de Argentina, y al sur y suroeste con las comunas de Los Lagos y Futrono.

El lago Panguipulli es alimentado por la desembocadura de dos importantes ríos, el río Enco y el río Llanquihue, este último nace del río Neltume y el río Fuy. El río Llanquihue desde el año 2012 presenta Didymo, por lo cual fue declarado por SERNAPESCA como zona de área de plaga.

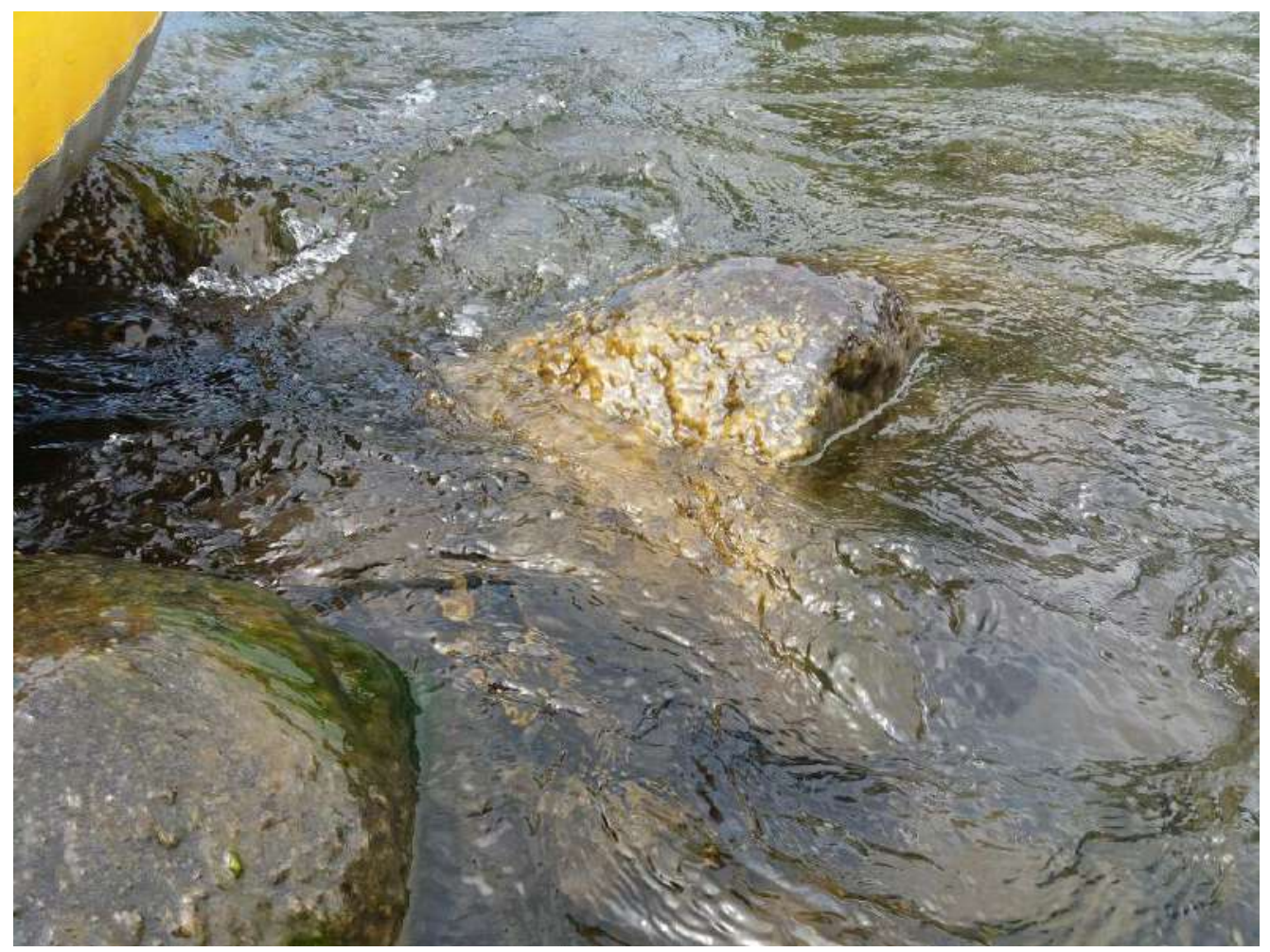

Figura 10: Moco de Roca “Didymo” en río Llanquihue.

Fuente: Inspector de Pesca Recreativa Amigos de Panguipulli.

De este modo, los deportistas y pescadores recreativos (profesionales) que visitan estas zonas, adquieren por normativa el Manual de Pesca Recreativa de SERNAPESCA, el cual describe acerca de esta plaga y los procedimientos de aseo y contención cuando se practica la pesca recreativa. Por el contrario, el potencial riesgo seguirá siendo la sociedad no informada (en 


\section{Felipe Ignacio Peña Galaz}

mayor escala), que practica la pesca ilegal y visita de forma esporádica el río, no poseen licencia de pesca recreativa, son, sin duda, el primer agente de dispersión de la plaga por microalga Didymosphenia geminata. En este escenario entonces, la destrucción del hábitat causado por la sociedad del riesgo, conlleva a que las invasiones biológicas figuren en la actualidad dentro de los principales factores que amenazan la biodiversidad a nivel mundial, donde el impacto es tal, que las invasiones biológicas se les considera uno de los problemas más importantes que afectan los ecosistemas (Quiroz et al. 2009).

\section{La Corporación de Adelanto Amigos de Panguipulli}

Producto del trabajo empírico en las macrozonas, se pudo obtener un hallazgo de gran relevancia para el estudio el cual fue encontrado en la ciudad de Panguipulli. Llama la atención en esta ciudad un entorno bastante ordenado, existen varios puntos de reciclaje y de clasificación de desechos, destacar en especial, un punto de limpieza para contención de Didymo, equipo ubicado en el centro de la ciudad al costado de la catedral. En este lugar se encuentra ubicado "La Corporación de Adelanto Amigos de Panguipulli”, agrupación que se conforma gracias a eventos culturales que son organizados por un grupo de personas que tienen como objetivo el cuidado del entorno territorial, los cuáles se desempeñan en cargos importantes en la comuna misma y en el Departamento de Proyectos de la Corporación Municipal de Panguipulli. Centran sus reuniones y proyectos del quehacer de la localidad y la protección del ecosistema, surge la idea de unirse para trabajar por el progreso de una localidad por la cual sienten un especial apego. Este grupo de adelanto, ha podido aplicar para el desconocimiento de ellos mismo un modelo conceptual de Inteligencia Territorial, han creado este grupo para promover la participación ciudadana, el emprendimiento y el cuidado del entorno. Según Guzmán (2013, p. 77) el desarrollo territorial supone que se lleve a cabo una gestión territorial del conocimiento, articulando redes que propicien la innovación y el emprendimiento alrededor de las posibilidades y potencialidades industriales y comerciales del territorio.

De esta forma, Amigos de Panguipulli en el contexto de Didymo, cumple el rol de difundir mediante material propio y donado por SERNAPESCA. Además fiscaliza con su propio personal, paralelo a los inspectores municipales y el personal de SERNAPESCA, multando a 


\section{Felipe Ignacio Peña Galaz}

quién no cumpla las normas establecidas para la pesca recreativa legal y actividades en sus ríos. De este modo en la zona del río Llanquihue, el Didymo está declarado "Plaga", pero se encuentra bajo control gracias a estas acciones territoriales.

Producto de estos fenómenos, para Girardot (2012) el territorio se debilitaría gradualmente frente a la explotación, exportación de bienes tradicionales y el turismo de la región, entonces se tiene que trabajar una visión sistémica, incluido un espacio geográfico, sus comunidades, sus representaciones y sus comportamientos, no es más que un enfoque multidisciplinario entre las partes, lo que se conoce en la literatura como Inteligencia Territorial.

La Inteligencia Territorial (IT) está directamente asociada a este tipo de fenómenos, en el redescubrimiento del territorio perdido, la IT plantea la necesidad e implementación de los planes territoriales estratégicos y que incentive la participación de los actores locales bajo el concepto de elementos inteligentes. La adquisición de mayor conocimiento es clave para controlar de forma óptima un territorio y la articulación de mejores políticas de desarrollo económico de un territorio, sólo es a través de la vinculación de investigadores ligados a cada ámbito, actores locales implicados en los procesos de cambio y comunidad territorial capaces de articular elementos de dinamización territorial, por consiguiente, esta definición gira en torno a dos ejes fundamentales, la investigación y la implicación de los actores en el proceso de cambio estructural y crecimiento del territorio (Parrilla y Pulido, 2017).

La literatura actual relacionada a Didymosphenia geminata demuestra que el problema medio ambiental y el efecto negativo en el turismo local que esta microalga genera, no emerge simplemente de las condiciones físicas (Broitman y Kreimer, 2017), sino que se configura mediante un juego de actores en el espacio público, (Hannigan, 2006).

\section{Conclusión}

De acuerdo al trabajo de observación se pudo evidenciar que los actores locales de estas Regiones tienen una baja participación frente a la plaga Didymo, este efecto sucede en mayor medida en Villarrica y Pucón, en estas localidades es interesante evidenciar a simple vista una masiva y excesiva difusión de oferta inmobiliaria y de explotación del turismo, no se aprecia en mayor medida más que algunos puntos información acerca de Didymo. Los organismos públicos como SERNAPESCA o consultorías privadas que apoyan la labora de monitoreo y prospección, 
no se evidencia que involucren en sus trabajos en terreno a los individuos (no científicos) locales o si lo hace es en una escala menor ya sea a pescadores, no obstante, sus visitas a las zonas afectadas por plaga son de carácter técnico, aquí se produce un quiebre social, ya que quiénes conviven todo el año con la plaga no están bien informados o no se les hace partícipe de esto, por lo tanto la educación en este contexto es muy baja, y el efecto económico es indirecto.

La divulgación acerca de Didymo geminata a través de redes sociales contiene información obsoleta o no mantiene una actualización constante en el quehacer de los organismos y las comunidades frente a la plaga, se encuentra información incluso de una data de 3 años de antigüedad. Por otra parte, si nos referimos a las papelerías dispuestas en algunos puntos de información turística y los soportes de las Campañas de Publicidad Social aplicados en carreteras locales, se han realizado trabajos de difusión con un bajo nivel estratégico comunicacional, según lo observado y extraído en el trabajo de campo, estas campañas no son entendidas por los usuarios del río, cuando se habla de "HAZLE CASO A LA NORMA" los individuos no entienden si se refiere a una persona de nombre "NORMA" o es la "NORMA" de limpieza, o incluso ambas, cuesta analizar ese título sobre todo para los usuarios que hacen uso del río de forma esporádica, peor aún, las piezas gráficas no son observadas por los usuarios cuando se observa en terreno el comportamiento de los individuos frente a las gráficas informativas.

Otro escenario se vive en la ciudad de Panguipulli, van por un camino distinto a las otras dos ciudades, producto del trabajo colaborativo y de integración que realizan grupos locales como Amigos de Panguipulli en conjunto con la comunidad, dejando a los distintos organismos públicos y privados como parte de sus alianzas estratégicas. Aquí se cumple en gran parte lo que se denomina en la literatura como "Inteligencia Territorial", producto de las acciones de integración de actores locales y el cuidado del medioambiente del lugar.

En este escenario, las campañas sociales no han sido efectivas para la población, no se evidencia un estudio analítico acerca de campañas sociales y difusión acerca de estas temáticas, la población en gran número no tiene conocimiento de la existencia y el problema que la microalga Didymo provoca en los ecosistemas acuáticos. Se debe replantear el diseño de las futuras campañas sociales, difusión e informativas para que no sean campañas únicamente estéticas y decorativas, la responsabilidad en ese sentido la tiene el organismo mandante, SERNAPESCA. 


\section{Bibliografía}

Bergey E., Cooper J.T \& Phillips B.C. (2009). Substrate characteristics affect colonization by the bloom-forming diatom Didymosphenia geminata. Aquatic Ecology 44: 33-40.

Broitman C., Kreimer P., (2017). Knowledge Production, Mobilization and Standardization in Chile’s HidroAysén Case. Springer Science+Business Media B.V. 2017.

Costa, J. (2004). La creciente autonomía de las marcas. La imagen de marca. Un fenómeno social (pág. 86). Málaga, España.

Díaz et al (2012). Manual para el Monitoreo e Identificación de la Microalga Bentónica Didymosphenia geminata. Subsecretaría de Pesca y Acuicultura. Recuperado de:

https://www.researchgate.net/publication/321849070_Manual_para_el_Monitoreo_e_Identificaci on_de_la_Microalga_Bentonica_Didymosphenia_geminata_Subsecretaria_de_Pesca_y_Acuicult ura (Revisado el 12.10.2018)

Hannigan, J., (2006). Environmental Sociology: A Social Constructivist Perspective. New York: Routledge \& Kegan Paul Books.

Herrera, C. (2012). Gestión ambiental en torno a la aparición del Didymo en el río Biobío, en la población de Troyo (Investigación en curso).

INE. (2002). Anuario de Turismo 2002. Recuperado de: www.ine.cl (Revisado el 12.10.2018) Jaramillo, A. (2015). Caracterización genética en muestras geográficamente aisladas de la diatomea invasora Didymosphenia geminata (Lyngbye) Schmidt M., 1899 en ríos de Chile Centro-Sur (Tesis licenciatura). Recuperado de:

http://cybertesis.uach.cl/tesis/uach/2015/fcj.37c/doc/fcj.37c.pdf (Revisado el 12.10.2018)

Kilroy, C. (2004). A new alien diatom, Didymosphenia geminata (Lyngbye) Schmidt: its biology, distribution, effects \& potential risks for New Zealand fresh waters. NIWA, Christchurch, New Zealand.

Kilroy, C., Biggs, B., Blair, N., Lambert, P., Jarvie, B., Dey, B., Robinson, K., \& Smale, D. (2005). Ecological studies of Didymosphenia geminata: National Institute of Water an Atmospheric Research, New Zealand, Client Report CHC2005-123, NIWA Project: MAF05505.

Kilroy, C., Lagerstedt, A., Davey, A. y Robinson, K. (2007). Studies on the survivability of the invasive diatom Didymosphenia geminata under a range of environmental \& chemical conditions. NIWA Client Report CHC2006-116. 
Lagerstedt, M. A. (2007). Didymosphenia geminata, an example of a biosecurity leak in New Zealand. Thesis in partial fulfilment of requirements for M.Sc. in Environmental Sciences, New Zealand: University of Canterbury

Orozco J. (2010). “Comunicación estratégica para campañas de publicidad social”. Pensar la Publicidad, volumen IV), $\mathrm{n}^{\mathrm{o}}$ 2, 2010. Recuperado de:

https://revistas.ucm.es/index.php/PEPU/article/download/PEPU1010220169A/15055

Parrilla J., Pulido J. (2017). Inteligencia Territorial y Turismo. Hacia la integración de un modelo de transformación económica. Estudios y perspectivas en turismo, volumen (26), $\mathrm{n}^{\mathrm{o}} 1$, enero 2017, páginas 1-21. Recuperado de:

http://www.scielo.org.ar/scielo.php?script=sci_arttext\&pid=S1851-17322017000100001

(Revisado el 12.10.2018)

Quiroz, C., Pauchard, A., Marticorena, A., Cavieres, L. (2009). Manual de Plantas Invasoras del Centro-Sur de Chile. Proyecto CONICYT PFB-23.

Reid, B. L., K. L. Hernández, M. Frangopulos, G. Bauer, M. Lorca, C. Kilroy, y S. Spaulding (2012). The invasion of the freshwater diatom Didymosphenia geminata in Patagonia: prospects, strategies, and implications for biosecurity of invasive microorganisms in continental waters. Conservation Letters 5:432-440.

Rivera, P., S. Basualto, y F. Cruces (2013). On the diatom Didymosphenia geminata (Lyngbye) M. Schmidt: its morphology and distribution in Chile. Gayana Botanica 70:154-158.

Salmonexpert (2016). Subpesca inicia talleres de difusión sobre plaga Didymo. Recuperado de: https://www.salmonexpert.cl/article/subpesca-inicia-talleres-de-difusion-sobre-plaga-didymo/ (Revisado el 12.10.2018)

SERNAPESCA (2012). ¿Qué es el Didymo? SERNAPESCA. Recuperado de:

http://didymo.sernapesca.cl/quees.php\#eng_(Revisado el 10.10.2018)

SERNATUR (2016). Anuario de Turismo 2016. Recuperado de: www.sernatur.cl (Revisado el 12.10.2018)

SERNATUR (2014-2018). Plan de acción Región de la Araucanía, sector turismo. Recuperado de: www.sernatur.cl (Revisado el 12.10.2018)

SUBPESCA/Amakaik-Ecohyd (2012). Informe Final "Prospección de Didymosphenia geminata en cuerpos de agua de la Zona Centro-Sur (Id 4728-40-R112)”. Octubre 2012- Abril 2013. 